Artículo

\title{
Tiene el amaranto el potencial agronómico para ser un fenómeno mundial como la quinua
}

\author{
Espitia Rangel Eduardo ${ }^{1}$ \\ Luisa Fernanda Sesma Hernández ${ }^{1}$ \\ Miriam Gabriela Valverde Ramos ${ }^{1}$ \\ Lucila González Molina ${ }^{1}$ \\ Diana Escobedo López ${ }^{1}$ \\ Miriam Jazmín Aguilar Delgado ${ }^{2 \S}$
}

${ }^{1}$ Campo Experimental Valle de México-INIFAP. Carretera los Reyes-Texcoco km 13.5, Coatlinchán, Texcoco, Estado de México, México. CP. 56250. (espitia.eduardo1957@gmail.com). ${ }^{2}$ Facultad de Ciencias Agrotecnológicas-Universidad Autónoma de Chihuahua. Calle La Presa de la Amistad núm. 2015, Barrio La presa, Ciudad Cuauthémoc, Chihuahua, México. CP. 31510.

§Autora para correspondencia: mjaguilar@uach.mx.

\section{Resumen}

El Amaranto y la quinua pertenecen a la familia Amaranthaceae caracterizada por tener especies que crecen en condiciones adversas, además de presentar altos contenidos de proteínas, ácidos grasos insaturados y vitaminas, además propiedades funcionales, que podrían ser una excelente opción para hacerle frente a los grandes problemas que aquejan al mundo. Debido a estas características la quinua se ha convertido en un fenómeno a nivel mundial y ya se cultiva en más de 100 países. La investigación fue planteada con el fin de determinar si el amaranto tiene el potencial agronómico para incrementar la superficie cultivada como sucedió con la quinua. Se estableció un experimento con tres variedades de quinua y tres variedades de amaranto en tres ambientes de los Valles Altos de México, bajo un diseño de bloques completos al azar con arreglo de tratamientos en parcelas divididas. Se evaluaron variables agronómicas, así como el rendimiento. Los resultados encontrados mostraron que el amaranto superó a la quinua en longitud de inflorescencia, ancho de inflorescencia, diámetro de tallo, peso hectolítrico y rendimiento, mientras que la quinua presentó valores más altos para diámetro de semilla y peso de mil semillas. Los genotipos Tlahuicole y L-145 de amaranto fueron los que observaron mejor comportamiento, seguidos por la variedad Suyana de quinua. El amaranto presenta características para ser un fenómeno a nivel mundial, tal como ha sucedido con la quinua.

Palabras clave: Amaranthus spp., Chenopodium quinoa, rendimiento, variedades.

Recibido: septiembre de 2021

Aceptado: noviembre de 2021 


\section{Introducción}

Para hacer frente a las grandes fuerzas que están moviendo a la sociedad como la desnutrición, problemas de salud pública y al cambio climático, es necesario utilizar especies vegetales con características que coadyuven a la solución de estos temas. Asimismo, en los programas de mejoramiento genético se deberá buscar variedades inteligentes que respondan a las condiciones ambientales que se presenten en la estación de crecimiento. Generalmente este tipo de variedades pueden crearse utilizando especies que por naturaleza puedan crecer en condiciones agroclimáticas adversas, tal es caso de las Cariophyllales conocidas por contener varias especies extremófilas.

Dentro de este grupo se tiene la familia Amaranthaceae que comprende entre otros los géneros Amaranthus y Chenopodium, caracterizados por tener especies que crecen en condiciones adversas, además de presentar altos contenidos de proteínas, ácidos grasos insaturados y vitaminas, además de propiedades funcionales, por tan podrían ser una excelente opción para hacerle frente a estos grandes problemas de salud, alimentación y ambientales que atraviesa el mundo. El amaranto es una planta herbácea anual que pertenece al género Amaranthus, predominantemente tropical, incluye cerca de 70 especies nativas de los trópicos y de las regiones templadas de todo el mundo; de las cuales 40 son de América y el resto pertenece a Australia, África, Asia y Europa.

Dentro del género se encuentran las especies A. cruentus, A. hypochondriacus y A. caudatus que son las más importantes para la producción de grano de amaranto (Espitia, 1992). La quinua es una planta del género Chenopodium, tiene amplia distribución mundial, con cerca de 250 especies (Zurita-Silva et al., 2014).

El cultivo del amaranto en México está poco desarrollado, pues se siembran de 3000 a 7000 ha anuales, fluctuando de acuerdo con la oferta y la demanda. Son muchas las limitantes que enfrenta este cultivo, tales como el bajo nivel tecnológico con el que se realiza. La mayoría de las siembras se realizan con variedades criollas de bajo rendimiento y características agronómicas desventajosas, como maduración tardía y plantas altas, variación en color de planta y semilla, entre otras. No existe tecnología de producción bien fundamentada para dosis de fertilización, fechas de siembra, densidades, control de plagas y enfermedades. Recientemente el amaranto fue incluido en la canasta básica, por lo tanto, para poder incrementar la superficie de siembra y de cosecha se hace necesario mejorar la tecnología utilizada hasta estos momentos.

El amaranto y la quinua además de su potencial agronómico de crecer en condiciones adversas son súper granos reconocidos dentro de los cultivos más prometedores para coadyuvar a conseguir la seguridad alimentaria y combatir la malnutrición (Präger et al., 2018). En los últimos años se ha incrementado el interés por estos granos, ricos en proteína y como fuente de compuestos nutraceuticos (Venskutonis y Kraujalis, 2013; Rastogi y Shuklam, 2013). Este interés obedece a la gran problemática actual asociada a enfermedades crónicas degenerativas, enfermedades isquémicas del corazón, cerebrovasculares y las crónicas del hígado, que se encuentran estrechamente ligadas a factores como malnutrición, sobrepeso y obesidad.

Por su lado, la quinua, es un cultivo de reciente introducción en México; sin embargo, en años recientes ha tomado un gran impulso debido al crecimiento de su consumo a nivel mundial. Este interés se debe a sus características agronómicas, nutritivas y nutraceuticas (Präger et al., 2018). 
Esta especie se ha cultivado en los Andes desde hace 5000 a 7000 años, adaptándose a altitudes cercanas a los $4000 \mathrm{msnm}$. La quinua y el amaranto poseen una alta eficiencia en el uso del agua e incluso puede producir rendimientos aceptables con lluvias de $200 \mathrm{~mm}$ anuales. También algunas variedades de quinua toleran condiciones de salinidad similares a las del agua de mar $\left(40 \mathrm{dS} \mathrm{m}^{-1}\right)$ superando en muchos casos la tolerancia de las especies cultivadas conocidas (Espitia 1994; ZuritaSilva et al., 2014).

La quinua es originaria de los Andes y está más adaptada a regiones entre 2500 a $4000 \mathrm{msnm}$ (Mujica et al., 1997), amaranto por su parte es originario de Mesoamérica y está más adaptado a regiones entre 0 a $2600 \mathrm{msnm}$. Aunque en los últimos tiempos estos cultivos han sido llevados a ambientes fuera de su rango de adaptación original. En relación con su potencial de rendimiento Bazile et al. (2014) reportaron rendimientos de 200 hasta $2050 \mathrm{~kg}^{-1}$ en un ensayo de 21 genotipos de quinua en diferentes países donde no se sembraba quinua en Europa y Asia. Chura et al. (2019) mencionan 2836.55 a $5099.18 \mathrm{~kg} \mathrm{ha}^{-1}$ para un grupo de seis líneas de quinua; mientras que, Zurita-Silva et al. (2014) reportan rendimiento de 400 a $4500 \mathrm{~kg} \mathrm{ha}^{-1}$.

Para amaranto en un ensayo similar (Mujica et al., 1997) se reporta rendimiento de 203 hasta 7208 $\mathrm{kg} \mathrm{ha}^{-1}$ para 12 genotipos en nueve países de América, en este caso fue en países donde se siembra amaranto. Los rendimientos reportados para México son muy variables y dependen de la variedad y de las condiciones de producción, por ejemplo, se mencionan de 2062 a $5274 \mathrm{~kg} \mathrm{ha}^{-1}$ (Espitia, 1992), de 3875 a $4583 \mathrm{~kg} \mathrm{ha}^{-1}$ (Mujica et al., 1997), de 950.7 a $2922.2 \mathrm{~kg} \mathrm{ha}^{-1}$ y de 1382 a 1 $668.7 \mathrm{~kg} \mathrm{ha}^{-1}$ (Ramírez, 2011) y de 1382 a $3439 \mathrm{~kg} \mathrm{ha}^{-1}$ (Ortiz et al., 2018).

En otras características de importancia agronómica también se reportan valores muy variables, por ejemplo, para la variable madurez se reportan de 132.5 a 161.38 días en 60 genotipos de las especies A. cruentus y A. hypochondriacus (Espitia et al., 1992), de 63.2 a 97.4 para 54 genotipos de A. hypochondriacus. Para altura de planta se reportan valores de 141.88 a $228.94 \mathrm{~cm}$ (Espitia et al., 1992) y de 81.3 a $148 \mathrm{~cm}$ (Tiwari et al., 2018). Otras características importantes son aquellas que expresan el tamaño y densidad de la semilla, a este respecto se reportan peso de mil semillas de 0.684 a $1.32 \mathrm{~g}$ (Tiwari et al., 2018) y peso hectolítrico de 82.51 a $83.51 \mathrm{~kg} \mathrm{~L}^{-1}$ (Espitia et al., 1992).

En la quinua es muy relevante el tamaño de la semilla pues de eso depende la comercialización y el uso, por lo que son relevantes las variables que expresan tamaño y peso de la semilla, a este respecto se reportan diámetro de semilla desde 1.2 hasta $2.5 \mathrm{~mm}$ (Zurita-Silva et al., 2014), mientras que Chura et al. (2019) reportan diámetros de 1.64 a $2.2 \mathrm{~mm}$. Los mismos autores refieren para el valor de peso de mil semillas de 2.09 a 3.8 g, Delgado et al. (2009) explicaron de 2.52 a $3.45 \mathrm{~g}$, mientras que, Curti et al. (2014) establecen valores de 2.2 a 3.5 g. Para madurez ZuritaSilva et al. (2014) presentaron valores que van desde 135 a 210 días, mientras que, Bhargava et al. (2007) reportan valores de 109.3 a 163.33 días a madurez. Para la variable altura de planta Delgado et al. (2009) demostraron valores de 111.23 a $176.65 \mathrm{~cm}$ y los mismos autores reportan para longitud de panoja valores de 24.03 a $37.25 \mathrm{~cm}$.

Recientemente la quinua se ha convertido en un fenómeno a nivel mundial, se cultiva en muchos países y con perspectivas de seguir creciendo (Bazile et al., 2016). El amaranto y la quinua son dos cultivos con muchas semejanzas morfológicas, nutritivas, de utilización y hasta de desarrollo histórico; nace la pregunta de por qué quinua ha crecido tanto en producción y consumo y el amaranto lejos de crecer de pronto hasta se ha reducido la superficie cultivada y en consecuencia 
su consumo. Por lo tanto, el objetivo del presente trabajo fue realizar una comparación agronómica del amaranto y la quinua para determinar el potencial que tiene cada especie en los Valles Altos del Centro de México.

\section{Materiales y métodos}

\section{Material genético}

Los genotipos del amaranto utilizados fueron L-145, Tlahuicole y Nutrisol de la especie $A$. hypochondriacus, mientras que Suyana, Tokyo y Suma fueron los genotipos de la quinua utilizados, los genotipos para representar las dos especies fueron escogidos en base a su ciclo y porte de planta y en el caso de quinua también se seleccionaron por presentar buena adaptación a las condiciones del Centro de México.

\section{Ambientes de evaluación}

Los ambientes de evaluación fueron dos en Santa Lucía de Prías, Texcoco (dos fechas de siembra) y Boyeros, Texcoco (Colonia Netzahualcóyotl), Estado de México en 2019. La fecha de siembra en Santa Lucía de Prías fue el 28 de mayo (la primera fecha) y 30 de junio (la segunda). Con la primera fecha se está simulando una fecha de siembra normal, cuando se establece el temporal; con la segunda se está buscando que la floración no coincida con las altas temperaturas que se presentan en julio. Para Colonia Netzahualcóyotl fue el 30 de junio, en este ambiente se está evaluando condiciones de salinidad.

\section{Manejo del cultivo}

El cultivo fue de temporal, sin fertilización, no hubo control de plagas y sólo se realizarron dos deshierbes con azadón a los 25 y 45 días despues de la siembra. A la quinua se le aplicó el fungicida Metalaxil (producto comercial: Ridomil Gold) $1 \mathrm{~L} \mathrm{ha}^{-1}$ a los 30 días de emergida la planta y Mancozeb a los 50 días de emergida la planta (producto comercial: Manzate) $1.5 \mathrm{~kg} \mathrm{ha}^{-1}$, con el fin de disminuir el efecto del Mildiu (Peronospora variabilis).

\section{Variables evaluadas}

Se evaluaron variables fenológicas como días a floración, días a madurez y periodo de llenado de grano (días), se contaron los días a cuando $50 \%$ de las plantas antesis y madurez y los días transcurrido de floración a madurez, respectivamente. Las variables de tamaño como diámetro de tallo $(\mathrm{cm})$, altura de planta a la floración $(\mathrm{cm})$, altura de planta a la madurez $(\mathrm{cm})$ y longitud de panoja $(\mathrm{cm})$, se midieron 10 plantas por parcela.

El diámetro de semilla se determinó midiendo 10 grupos de 10 semillas de cada parcela, con un vernier digital (Stainless Hardened). Peso hectolítrico $\left(\mathrm{kg} \mathrm{hl}^{-1}\right)$ se determinó pesando un volumen conocido de semilla y se extrapoló al peso de $100 \mathrm{~L}$. Para el peso de mil granos (g), se contaron y pesaron cinco grupos de 100 granos de cada parcela y se extrapoló a mil granos y el rendimiento de grano $\left(\mathrm{kg} \mathrm{ha}^{-1}\right)$ se determinó a partir del rendimiento de la parcela útil y se extrapoló a 1 ha. 


\section{Diseño experimental, unidad experimental y análisis estadístico}

Los genotipos se sembraron bajo un diseño experimental de bloques al azar con cuatro repeticiones y un arreglo de tratamientos en parcelas divididas, la parcela grande correspondió a las especies y los genotipos a la parcela chica. La parcela experimental consistió en tres surcos a $0.8 \mathrm{~m}$ de separación y $5 \mathrm{~m}$ de largo y la parcela útil consistió en tres $\mathrm{m}$ del surco central. Para el análisis estadístico se utilizó Proc Glm del Sas y para la comparación de medias la prueba de Tukey (0.05).

\section{Resultados y discusión}

Se encontraron diferencias altamente significativas en la mayoría de las variables para ambientes, excepto para diámetro de semilla y rendimiento por día (Cuadro 1). Para especies se encontraron diferencias significativas en todas las variables excepto en altura de planta. Para genotipos dentro de especies también se obtuvieron diferencias significativas excepto en longitud de inflorescencia, ancho de inflorescencia y diámetro de tallo. Para la interacción especies por ambiente, se obtuvieron diferencias significativas para la mayoría las variables excepto para altura de planta, longitud de inflorescencia y diámetro de semilla. Lo que indica una respuesta diferencial de las especies a estas fuentes de variación coincidiendo con lo reportado anteriormente (Präger et al., 2018; Tiwari et al., 2018).

Cuadro 1. Cuadrados medios para las variables estudiadas entre especies en tres ambientes de México. V 2019.

\begin{tabular}{ccccccc}
\hline Variable/FV (gl) & Loc (2) & $\begin{array}{c}\text { Rep (loc) } \\
(9)\end{array}$ & Esp (1) & $\begin{array}{c}\text { Var (esp) } \\
(4)\end{array}$ & Loc*esp (2) & $\begin{array}{c}\text { Error } \\
(53)\end{array}$ \\
\hline Emergencia de inflorescencia & $83.6^{* *}$ & 1.94 & $3186.68^{* *}$ & $297.01^{* *}$ & $200.47^{* *}$ & 5.12 \\
(días) & $36.3^{* *}$ & 2.27 & $3486.13^{* *}$ & $398.67^{* *}$ & $156.29^{* *}$ & 5.32 \\
Días a floración & $8553.6^{* *}$ & 0.65 & $826.89^{* *}$ & $481.22^{* *}$ & $4311.01^{* * *}$ & 7.83 \\
Días a madurez & $7712.3^{* *}$ & 0.9 & $7708.68^{* *}$ & $335.89^{* *}$ & $4085.18^{* *}$ & 12.9 \\
Periodo de llenado de grano & $60601.4^{* *}$ & 197.42 & $57.96 \mathrm{~ns}$ & $1081.44^{* *}$ & $30607.65^{* *}$ & 194.16 \\
Altura de planta (cm) & $1698.2^{* *}$ & 355.15 & $2678.63^{* *}$ & $147.44 \mathrm{~ns}$ & $1454^{* *}$ & 201.41 \\
Longitud de la inflorescencia (cm) & $564.2^{* *}$ & 20.73 & $598.64^{* *}$ & $5.52 \mathrm{~ns}$ & $420.31^{* *}$ & 13.14 \\
Ancho de la inflorescencia (cm) & $85.2^{* *}$ & 5.12 & $1146.91^{* *}$ & $11.66 \mathrm{~ns}$ & $104.86^{* *}$ & 6.8 \\
Diámetro de tallo (mm) & $0.05 \mathrm{~ns}$ & 1 & $1081.6^{* *}$ & $0.01^{* *}$ & $0.07 \mathrm{~ns}$ & 0.02 \\
Diámetro de semilla (mm) & $0.1^{* *}$ & 0.01 & $60.09^{* *}$ & $0.34^{* *}$ & $0.11^{* *}$ & 0.01 \\
Peso de mil semillas (g) & $22.6^{* *}$ & 0.36 & $6595.6^{* *}$ & $63.39^{* *}$ & $27.63^{* *}$ & 3.71 \\
Peso hectolítrico (kg) & $10170752^{* *}$ & 3594596.9 & $10957044^{* *}$ & $6110264^{* *}$ & $11030525^{* *}$ & 967667 \\
Rendimiento (kg ha $\left.{ }^{-1}\right)$ & & & & &
\end{tabular}

${ }^{* *}=$ diferencias significativas; $\mathrm{ns}=$ diferencias no significativas.

En el Cuadro 2 se presenta la comparación de medias entre especies. En las variables fenológicas el amaranto presentó mayor número de días a emergencia de panoja y días a floración. Sin embargo, para periodo de llenado de grano y madurez la quinua presentó mayor número de días. 
A pesar de que en la quinua emerge primero la inflorescencia y se inicia la floración, esta especie tarda más tiempo en llegar a la maduración, esto es debido al mayor periodo de llenado de grano, pero aun así la quinua es menos tardía que en sus lugares de origen. En Bolivia y Perú madura hasta en más 200 días (Rojas et al., 2013; Chura et al., 2019), esto debido a que ahí crece cerca de los 4000 msnm, mientras que en México se sembró a 2250 msnm.

Cuadro 2. Comparación de medias de variables estudiadas entre especies en tres ambientes de México. V 2019.

\begin{tabular}{cccc}
\hline Variable/especie & $\begin{array}{c}\text { Amaranthus } \\
\text { hypochondriacus }\end{array}$ & $\begin{array}{c}\text { Chenopodium } \\
\text { quinoa }\end{array}$ & DSH \\
\hline Días a inflorescencia & $77.72 \mathrm{a}$ & $64.41 \mathrm{~b}$ & 1.0675 \\
Días a floración & $90.91 \mathrm{a}$ & $77 \mathrm{~b}$ & 1.0882 \\
Días a madurez & $146.63 \mathrm{~b}$ & $153.41 \mathrm{a}$ & 1.3201 \\
Periodo de llenado de grano (días) & $55.72 \mathrm{~b}$ & $76.41 \mathrm{a}$ & 1.6939 \\
Altura de planta (cm) & $234.31 \mathrm{a}$ & $236.1 \mathrm{a}$ & 6.5721 \\
Longitud de la inflorescencia (cm) & $58.9 \mathrm{a}$ & $46.7 \mathrm{~b}$ & 6.6937 \\
Ancho de la inflorescencia (cm) & $20.21 \mathrm{a}$ & $14.44 \mathrm{~b}$ & 1.7094 \\
Diámetro de tallo (mm) & $22.21 \mathrm{a}$ & $14.2 \mathrm{~b}$ & 1.2392 \\
Diámetro de semilla (mm) & $1.1 \mathrm{~b}$ & $1.92 \mathrm{a}$ & 0.0253 \\
Peso de mil semillas $(\mathrm{g})$ & $0.81 \mathrm{~b}$ & $2.67 \mathrm{a}$ & 0.0511 \\
Peso hectolítrico $(\mathrm{kg})$ & $81.71 \mathrm{a}$ & $62.2 \mathrm{~b}$ & 0.9228 \\
Rendimiento $\left(\mathrm{kg} \mathrm{ha}{ }^{-1}\right)$ & $3825.8 \mathrm{a}$ & $3056.9 \mathrm{~b}$ & 467.44 \\
\hline
\end{tabular}

Medias en hileras con las mismas letras son estadísticamente iguales.

En las variables de tamaño el amaranto presenta ventaja en longitud y diámetro de la inflorescencia y diámetro de tallo, mientras que ambas especies presentaron la misma altura de planta. En las variables de semilla la quinua presentó mayor diámetro de semilla, mayor peso de mil granos, por consiguiente, el amaranto presenta mayor peso hectolítrico; cabe aclarar, que este valor es de la quinua sin beneficiar; es decir, todavía con el pericarpio adherido a la semilla. En rendimiento el amaranto presentó mayor rendimiento de grano por día y mayor rendimiento de grano. Esto puede explicarse porque los sitios de evaluación son lugares donde tradicionalmente se siembra amaranto (Espitia, 1992; Espitia, 1994) y la quinua es introducida en ellos.

De las características más contrastadas es el tamaño de la semilla. El diámetro de semilla de las tres variedades de quinua evaluadas varió de 1.8 a $1.9 \mathrm{~mm}$, quedando clasificado como grano grande (Murphy et al., 2019). El tamaño de grano también está relacionado con la utilización, granos grandes mayores a $1.8 \mathrm{~mm}$ son utilizados para consumo graneado, que es la forma más extendida a nivel mundial (INDECOPI, 2014). La quinua por su mayor tamaño de semilla presenta menos problemas de manejo agronómico, pues germina a los tres o cuatro días, mientras que el amaranto germina a los ocho o diez días, por lo anterior la quinua presenta mejor emergencia de plántulas. Los valores aquí obtenidos para peso de mil granos (2.67 g) es comparable con los reportados (2.3 a 3.2 g) en Sudamérica (Curti et al., 2014). 
En relación con el rendimiento por día el amaranto presentó $25.42 \mathrm{~kg}$ por día casi cinco más que la quinua; mientras que en rendimiento de grano también el amaranto presentó un rendimiento mayor que la quinua, casi $800 \mathrm{~kg} \mathrm{ha}^{-1}$ más en promedio de las tres variedades de cada cultivo. Los valores de rendimiento para quinua en los tres ambientes del presente estudio son superiores a los reportados en un ensayo para 12 genotipos en el norte de Argentina (Curti et al., 2014), reportan 654 a $1703 \mathrm{~kg} \mathrm{ha}^{-1}$ en ambientes expuestos a sequía.

En general se puede establecer que tanto el amaranto como la quinua presenta potencial para ser cultivada en las condiciones evaluadas. Es probable que quinua por su origen presente mejor comportamiento conforme se cultive en ambientes más cercanos a los $3000 \mathrm{msnm}$ (Zurita-Silva et al. 2014). Por el contario a medida que se va bajando en altitud es probable que quinua presente problemas, especialmente si se presentan temperaturas de $30{ }^{\circ} \mathrm{C}$ durante la floración, pues esto ocasiona esterilidad ya que se reduce la formación de grano (Bertero, 2013).

En el Cuadro 3 se presentan las medias para las tres variedades de amaranto estudiadas en el presente experimento. Las líneas experimentales Tlahuicole y L-145 fueron las que presentaron mayor rendimiento (4 $097 \mathrm{~kg} \mathrm{ha}^{-1}$ ), mientras que Nutrisol, que es una variedad comercial rindió un poco menos que una tonelada. Cabe mencionar, que esta última mostró una madurez 15 días menos que las dos primeras al igual que el resto de las variables fenológicas que están correlacionadas inversamente con el rendimiento (Espitia et al., 1992).

Cuadro 3. Comparación de medias de variables estudiadas en tres variedades de amaranto en tres ambientes de México. V 2019.

\begin{tabular}{ccccc}
\hline Variable/variedad & Tlahuicole & L-145 & Nutrisol & DSH \\
\hline Días a inflorescencia & $80 \mathrm{a}$ & $78.4 \mathrm{a}$ & $74.7 \mathrm{~b}$ & 2.105 \\
Días a floración & $93.4 \mathrm{a}$ & $91.9 \mathrm{a}$ & $87.4 \mathrm{~b}$ & 2.386 \\
Días a madurez & $151.9 \mathrm{a}$ & $151.2 \mathrm{a}$ & $136.7 \mathrm{~b}$ & 1.439 \\
Periodo de llenado de grano & $59.3 \mathrm{a}$ & $58.5 \mathrm{a}$ & $49.3 \mathrm{~b}$ & 2.485 \\
Altura de planta $(\mathrm{cm})$ & $244.9 \mathrm{a}$ & $229.4 \mathrm{~b}$ & $228.5 \mathrm{~b}$ & 14.062 \\
Longitud de la inflorescencia (cm) & $60.4 \mathrm{a}$ & $56 \mathrm{ab}$ & $60.2 \mathrm{~b}$ & 4.408 \\
Ancho de la inflorescencia $(\mathrm{cm})$ & $20.7 \mathrm{a}$ & $19.4 \mathrm{a}$ & $20.5 \mathrm{a}$ & 2.531 \\
Diámetro de tallo $(\mathrm{mm})$ & $23 \mathrm{a}$ & $20.8 \mathrm{~b}$ & $22.8 \mathrm{ab}$ & 2.082 \\
Diámetro de semilla $(\mathrm{mm})$ & $1.14 \mathrm{a}$ & $1.13 \mathrm{a}$ & $1.11 \mathrm{a}$ & 0.051 \\
Peso de mil semillas $(\mathrm{g})$ & $0.78 \mathrm{~b}$ & $0.85 \mathrm{ab}$ & $0.81 \mathrm{~b}$ & 0.042 \\
Peso hectolítrico $\left(\mathrm{kg}^{-}\right)$ & $81.57 \mathrm{a}$ & $81.5 \mathrm{a}$ & $81.95 \mathrm{a}$ & 6.369 \\
Rendimiento $\left(\mathrm{kg} \mathrm{ha}^{-1}\right)$ & $4097 \mathrm{a}$ & $4412 \mathrm{a}$ & $2968 \mathrm{~b}$ & 1155.5 \\
\hline
\end{tabular}

Medias en hileras con las mismas letras son estadísticamente iguales.

En el Cuadro 4 refiere a la comparación de medias para las tres variedades de quinua evaluadas, Suyana presentó mayor rendimiento, mayor tamaño y peso de semilla y mayor tamaño de planta, rindió más que la variedad de amaranto Nutrisol y apenas $300 \mathrm{~kg}$ menos que las mejores variedades de amaranto. No se presenta la información de Mildiu (Peronospora variabilis), pero esta variedad fue la que presentó menor incidencia de esta enfermedad, que es uno de los factores adversos para 
la producción de quinua a nivel mundial (Murphy et al., 2019). De igual manera el contenido de saponinas es un aspecto importante en la producción de quinua (Zurita-Silva et al., 2014), Suma fue la variedad con menor contenido seguida de Suyana.

Cuadro 4. Comparación de medias de variables estudiadas en tres variedades de quinua en tres ambientes de México. V 2019.

\begin{tabular}{ccccc}
\hline Variable/variedad & Suyana & Tokio & Suma & DSH \\
\hline Días a inflorescencia & $60.9 \mathrm{~b}$ & $60.4 \mathrm{~b}$ & $71.9 \mathrm{a}$ & 1.42 \\
Días a floración & $74.3 \mathrm{~b}$ & $71.1 \mathrm{c}$ & $85.5 \mathrm{a}$ & 1.43 \\
Días a madurez & $151.9 \mathrm{~b}$ & $151.9 \mathrm{~b}$ & $156.4 \mathrm{a}$ & 1.03 \\
Periodo de llenado de grano & $77.5 \mathrm{~b}$ & $80.7 \mathrm{a}$ & $70.9 \mathrm{c}$ & 1.79 \\
Altura de planta $(\mathrm{cm})$ & $247.2 \mathrm{a}$ & $232.3 \mathrm{ab}$ & $228.7 \mathrm{a}$ & 15.5 \\
Longitud de la inflorescencia (cm) & $51.6 \mathrm{a}$ & $44.7 \mathrm{a}$ & $43.7 \mathrm{a}$ & 19.4 \\
Ancho de la inflorescencia (cm) & $15 \mathrm{a}$ & $13.7 \mathrm{a}$ & $14.5 \mathrm{a}$ & 4.84 \\
Diámetro de tallo $(\mathrm{mm})$ & $14.9 \mathrm{a}$ & $13.8 \mathrm{a}$ & $13.7 \mathrm{a}$ & 3.37 \\
Diámetro de semilla $(\mathrm{mm})$ & $1.9 \mathrm{a}$ & $1.9 \mathrm{a}$ & $1.8 \mathrm{a}$ & 0.04 \\
Peso de mil semillas $(\mathrm{g})$ & $2.9 \mathrm{a}$ & $2.6 \mathrm{~b}$ & $2.4 \mathrm{c}$ & 0.12 \\
Peso hectolítrico $(\mathrm{kg})$ & $63.2 \mathrm{a}$ & $64.82 \mathrm{a}$ & $58.5 \mathrm{a}$ & 6.86 \\
Rendimiento $\left(\mathrm{kg} \mathrm{ha}^{-1}\right)$ & $3800.7 \mathrm{a}$ & $2556.3 \mathrm{~b}$ & $2791.7 \mathrm{~b}$ & 733 \\
\hline
\end{tabular}

Medias en hileras con las mismas letras son estadísticamente iguales.

Por el comportamiento de ambas especies se ve claramente una ligera ventaja para el amaranto, pues presenta mayor rendimiento y menor ciclo de cultivo. La quinua por su parte presenta una gran ventaja que es el tamaño de grano, lo cual facilita el manejo agronómico del cultivo. Si vemos de manera más concreta a nivel de variedades individuales la variedad de quinua Suyana tiene muy buen comportamiento, comparable con las de amaranto.

Una desventaja de la quinua sería el perigonio de la semilla, en el cual se encuentran las saponinas, esto obliga a que en el manejo de postcosecha la semilla se tenga que escarificar o perlar para eliminar la 'cáscara' y luego lavarse para eliminar todas las saponinas (Zurita-Silva et al., 2014). Estos procesos de manejo de postcosecha encarecen el grano de quinua. Por el contrario, el amaranto se cosecha, se limpia y está listo para utilizarse, por no tener perigonio. Por lo anterior y de acuerdo con el objetivo planteado se puede establecer que amaranto posee las características agronómicas para expandir su cultivo, de manera similar como ha sucedido con la quinua. Las limitantes del amaranto tal vez sean de otra índole, como voluntad política, por estar en una región más occidentalizada no se ha popularizado el consumo local tanto como la quinua en sus lugares de origen.

Como se puede desprender de la magnitud de los cuadrados medios presentados en el análisis de varianza (Cuadro 1), la variación debida a la interacción especie x ambiente es comparable a la variación debida a las fuentes de variación ambientes y especies. En las Figuras 1 y 2 se presentó el comportamiento de las especies a través de los ambientes evaluados. Se puede observar que hubo cambios en magnitud y en orden en variables como días a floración, periodo de llenado de grano, longitud de panoja (Figura 1), diámetro de tallo y rendimiento (Figura 2). 

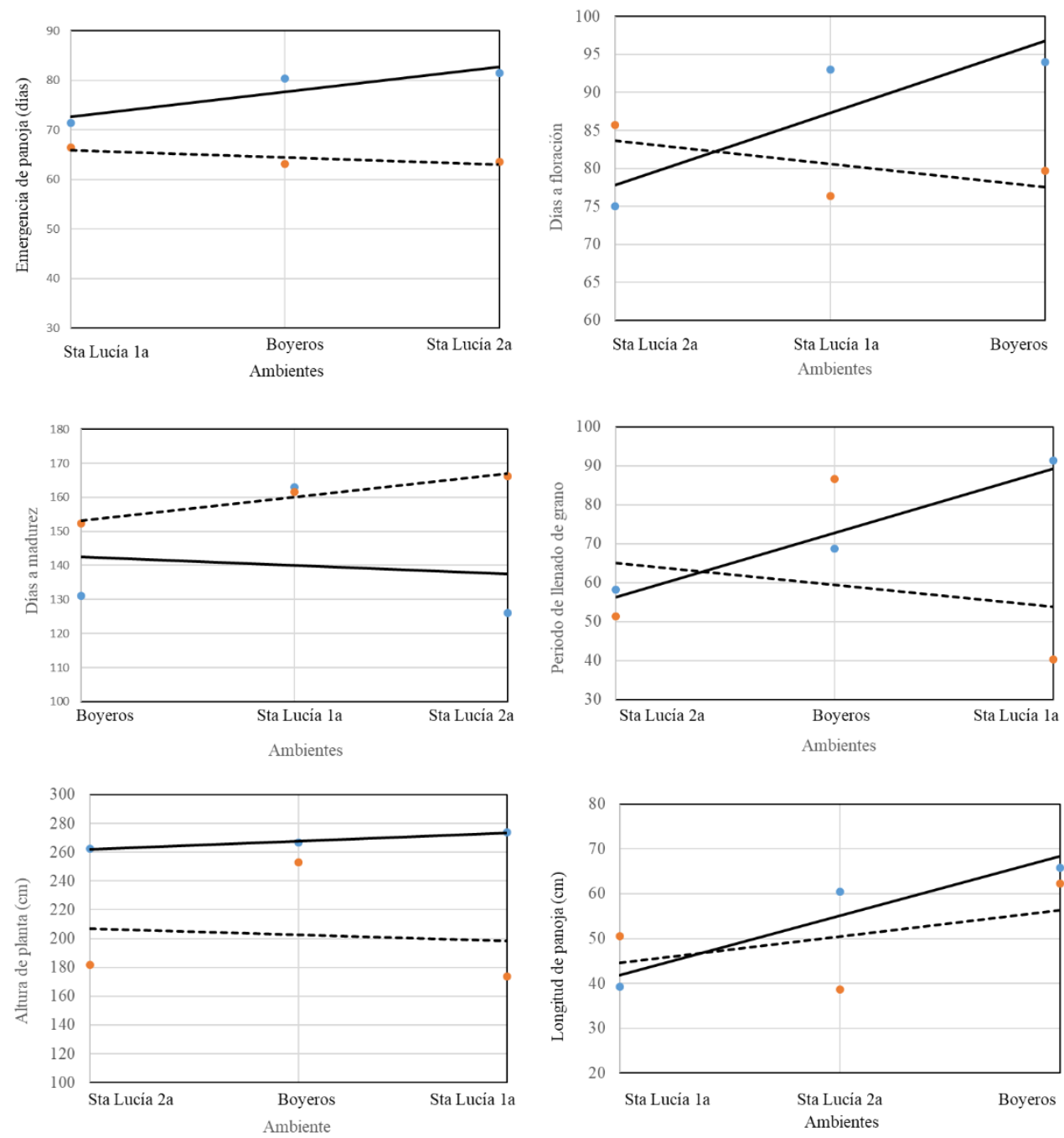

Figura 1. Interacción especie por ambiente de seis variables de la comparación de tres genotipos de amaranto (línea continua) y tres genotipos de quinua (línea discontinua) en tres ambientes. P-V 2019.

En las variables asociadas a la fenología hubo variación a través de ambientes, en días a floración y periodo de llenado de grano hubo cambios en orden y magnitud al cambiar de ambiente, tendiendo el amaranto a presentar mayores valores de estas variables al mejorar el ambiente, mientras que en emergencia de panoja el amaranto se mantuvo por encima de la quinua en los tres ambientes de prueba, caso contrario sucedió en días a madurez, estos resultados concuerdan con lo reportado anteriormente (Espitia, 1992; De Santis et al., 2018).

En variables de tamaño longitud de panoja (Figura 1) y diámetro de tallo (Figura 2) se presentaron cambios de magnitud y de orden, presentando valores más altos el amaranto, en altura de planta (Figura 1) y ancho de panoja (Figura 2) se obtuvieron cambios de magnitud, siendo el amaranto el que presentó los valores más altos a través de los ambientes evaluados, estos resultados concuerdan con lo reportado con anterioridad (Tiwari et al., 2018; Thiam et al., 2021). 

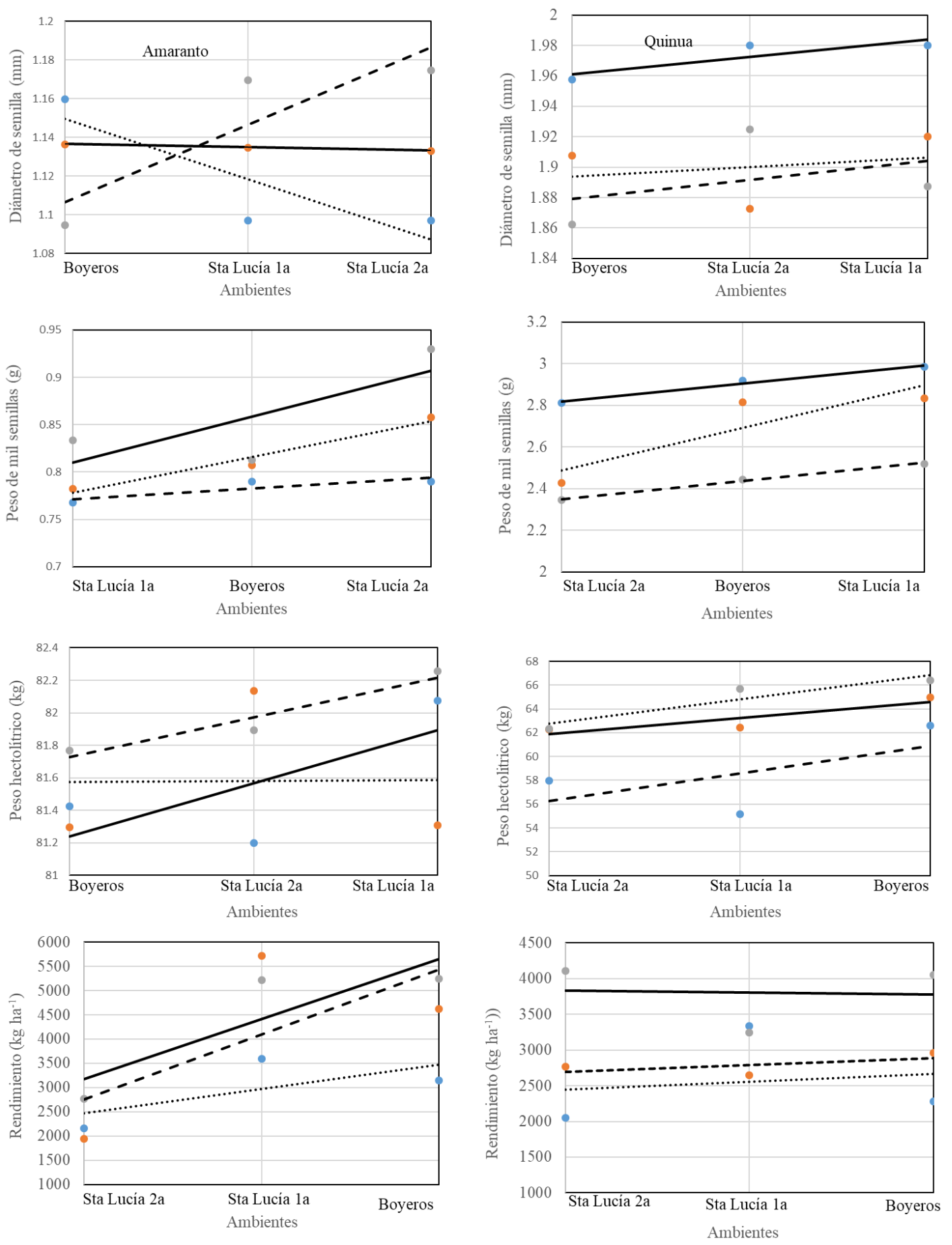

Figura 2. Interacción genotipo por ambiente del rendimiento de la comparación de tres genotipos de amaranto (línea continua Tlahuicole, línea discontinua L-145 y línea punteada Nutrisol) y tres genotipos de quinua (línea continua Suyana, línea discontinua Tokio y línea punteada Suma) en tres ambientes. P-V 2019.

Para las variables relacionadas con la semilla se obtuvieron cambios de magnitud en diámetro de semilla, peso de mil granos y peso hectolítrico (Figura 2), presentando la quinua el mayor tamaño de semilla, aunque para diámetro de semillas la interacción ambiente x especie no fue significativa, 
lo cual indica que esta variable está controlada principalmente por efectos genéticos esto se queda demostrado ya que en el análisis de varianza solo la fuente de variación especies fue significativa, mientras que ambientes y ambientes $\mathrm{x}$ especie fueron no significativas. Indicando esto que la significancia en especies se debe a la expresión del diámetro de semilla tan diferente entre las dos especies, ya que la quinua presenta un diámetro de semilla casi al doble que el amaranto.

Dicha diferencia también es notable en peso de 1000 semillas, pues la quinua presenta un valor tres veces mayor que el amaranto. Caso contrario sucede en peso hectolítrico donde amaranto presentó valor de $81.71 \mathrm{~kg}$ y la quinua de $62.2 \mathrm{~kg}$, que era de esperarse por menor tamaño de semilla que da mayor densidad y menos espacios entre semillas. Estos resultados concuerdan con lo reportado por Thiam et al. (2021) para quinua y para amaranto (Espitia,1992; Tiwari et al., (2018).

Para rendimiento se presentaron cambios de orden y cambios de magnitud; la quinua presentó mejor rendimiento en el ambiente más desfavorable que fue Santa Lucía de Prías segunda Fecha, mientras que, amaranto presentó mejor rendimiento en Boyeros y en Santa Lucía de Prías primer fecha, indicando esto que el comportamiento de amaranto mejora conforme mejora el ambiente (Tiwari et al., 2018), quinua por el contrario presentó una ligera disminución, esto debido tal vez a la incidencia de mildiu (Peronospora variabilis) en fecha de siembra más tempranas, esta enfermedad es una de las limitantes a nivel mundial para la producción de quinua (Khalifa and Thabet, 2018).

Que el amaranto haya resultado con una ventaja en rendimiento resultan normal, ya que las evaluaciones se realizaron en su ambiente de adaptación natural, mientras que la quinua se está introduciendo. Por su resistencia a bajas temperaturas la quinua en México se puede utilizar en la rotación de cultivos en zonas cerealeras donde se produce, cebada, trigo y avena ya que es una planta de hoja ancha lo que permitiría, reducir plagas y enfermedades de cereales al ser incorporada al patrón de cultivos de estas regiones. También se mejoraría el suelo ya que el rastrojo de quinua se incorporaría en su totalidad, la paja de los cereales es esquilmada en pacas, incrementando el contenido de materia orgánica y la capacidad de intercambio catiónico.

En los tres ambientes evaluados puede verse que en general el amaranto presentó mejor comportamiento que la quinua; sin embargo, la variedad Suyana de quinua presentó un comportamiento muy similar a las mejores variedades de amaranto (Tlahuicole y L-145), por lo cual se puede establecer que seleccionando los genotipos adecuados se pueden tener buenos rendimientos de ambos cultivos.

Una de las características para la comercialización y consumo de la quinua es el tamaño de la semilla, la norma técnica peruana reporta que los granos clasificados como grandes son aquellos mayores a $1.7 \mathrm{~mm}$, medianos entre 1.7 y 1.4 y granos pequeños menores a $1.4 \mathrm{~mm}$ de diámetro (INDECOPI, 2014), los resultados obtenidos con los genotipos evaluados fueron satisfactorios ya que presentaron grano grande. Una desventaja de la quinua es el hecho de tener perigonio, que es donde se encuentran las saponinas, compuestos que dan un sabor amargo, éstos deben ser eliminados por métodos físicos como la escarificación, métodos húmedos como el lavado o la combinación de ambos (Zurita-Silva et al., 2014), el amaranto no presentó perigonio y la semilla está lista para utilizarse cuando se cosecha, esta es una ventaja del amaranto. Agronómicamente se puede establecer que ambos cultivos tienen un buen potencial; es decir, se complementan ya que el amaranto se adapta más a climas templados a calientes y la quinua de templados a fríos. 


\section{Conclusiones}

El amaranto en general presentó mejor comportamiento en los ambientes evaluados que la quinua. Amaranto superó a la quinua en longitud de inflorescencia, ancho de inflorescencia, diámetro de tallo, peso hectolítrico y rendimiento; mientras que, la quinua presentó valores más altos para diámetro de semilla y peso de mil semillas. Los genotipos Tlahuicole y L-145 de amaranto fueron los que presentaron mejor comportamiento, seguidos por la variedad Suyana de quinua. El amaranto observó las características para ser un fenómeno a nivel mundial, tal como ha sucedido con la quinua.

\section{Literatura citada}

Bazile, D. 2014. The dynamics of the global expansion of quinoa growing in view of its high biodiversity. In: Bazile, D.; Bertero, D. y Nieto, C. (Ed.). Estado del arte de la quinua en el mundo en 2013. FAO. Santiago de Chile y CIRAD, Montpellier, Francia. $42-55$ pp. https://agritrop.cirad.fr/575493/1/document_575493.pdf.

Bazile, D. C.; Pulvento, A.; Verniau, M. S.; Al-Nusairi, J.; Breidy, L.; Hassan, M. I.; Mohammed, O.; Mambetov, M. O.; Sepahvand, N. A.; Shams, A.; Souici, D. and Padulosi, S. 2016. Worldwide evaluations of quinoa: preliminary results from post international year of quinoa FAO projects in nine countries. Frontiers in Plant Sci. 7(1):1-18. doi.org/10.3389/fpls.2016.00850.

Bertero, D. 2013. Environmental control of development. In: Bazile, D.; Bertero, D. y Nieto, C. (Ed.). Estado del arte de la quinua en el mundo en 2013. FAO. Santiago de Chile y CIRAD. Montpellier, Francia. 120-130 pp.

Bhargava, A.; Shukala, S. and Ohri, D. 2007. Genetic variability and interrelationship among various morphological and quality traits in quinoa (Chenopodium quinoa Willd.). Field Crops Res. 101(1):104-116. doi.org/10.3390/plants10040714.

Chura, E.; Mujica, A.; Haussmann, B.; Smith, K.; Flores, S. and Flores, A. L. 2019. Agronomic characterization of quinoa (Chenopodium quinoa Willd.) progeny from close and distant self-fertilized s5 simple crosses. Ciencia e Investigación Agraria. 46(2):154-165. doi 10.7764/rcia.v46i2.2142.

Curti, R. N.; De la Vega, A. J.; Andrade, A. J.; Bramardi, S. J. and Bertero, H. D. 2014. Multienvironmental evaluation for grain yield and its physiological determinants of quinoa genotypes across Northwest Argentina. Field Crops Res. 166(1):46-57. doi.org/10.1016/j.fcr.2014.06.011.

De Santis, G.; Ronga, D.; Caradonia, F.; Ambrosio, T. D.; Troisi, J.; Rascio, A.; Fragosso, M.; Pechioni, N. and Rinaldi, M. 2018. Evaluation of two groups of quinoa (Chenopodium quinoa Willd.) accesions with different seed colours for adaptation to de Mediterranean environment. Crops and Pasture Sci. 69(6):1264-1275. https://doi.org/10.1071/CP18143.

Delgado A. I. P.; Palacios, C. J. H. y Betancourt, C. G. 2009. Evaluación de 16 genotipos de quinua dulce (Chenopodium quinoa Willd.) en el municipio de Iles, Nariño, Colombia. Agron. Colomb. 27(2):159-167.

Espitia, R. E. 1992. Amaranth germplasm development and agronomic studies in México. Food Reviews International. 8(1):71-86. doi.org/10.1080/87559129209540930.

Espitia, R. E.; Miranda, C. S. y Castillo, G. F.1992. Variabilidad genética e interrelaciones del rendimiento y sus componentes en alegría (Amaranthus spp.). Agrociencia. 3(4):83-98. 
Espitia, R. E. 1994. Breeding of grain amaranth. In: Paredes-López, O. (Ed.) Amaranth: biology, chemistry and technology. CRC press, Boca Raton, USA. 23-38 pp.

INDECOPI. 2014. Compendio de normas técnicas peruanas: Quinua y Cañihua. NTP 205.062.2014. 1-25 pp.

Khalifa, W. and Thabet, M. 2018. Variation in downy mildew (Peronospora variabilis Gäum) resistance of some quinoa (Chenopodium quinoa Willd.) cultivars under Egyptian conditions. Middle East Journal of Agriculture Research. 7(2):671-682. https://www.researchgate.net/profile/Marian-Thabet/publication/328253874.

Mujica, S. A.; Berti, D. M. e Izquierdo, J. 1997. El cultivo del amaranto (Amaranthus spp.): producción mejoramiento genético y utilización. FAO. Roma, Italia. 145 p.

Murphy, K. M.; Matanguihan, J. B.; Fuentes, F. F.; Gómez-Pando, L. R.; Jellen, E. N.; Maughan, P. J. and Jarvis, D. E. 2019. Quinoa breeding and genomics. Plant Breeding Reviews. 42(1):257-320. https://mosaic.messiah.edu/edbooks/64.

Ortiz-Torres, E.; Argumedo-Macías A.; García-Perea, H.; Meza-Varela, R.; Bernal-Muñoz, R. y Taboada-Gaytán, O. R. 2018. Rendimiento y volumen de expansión de grano de variedades mejoradas de amaranto para valles altos de Puebla. Rev. Fitotec. Mex. 41(3):291-300.

Präger, A.; Munz, S.; Nkebiwe, P. M.; Mast, B. and Graeff-Hooninger, S. 2018. Yield and quality characteristics of different quinoa (Chenopodium quinoa Willd.) cultivars grown under field conditions in Southwestern Germany. Agronomy. 8(197):1-19. doi:10.3390/ agronomy8100197.

Ramírez, V. M. L.; Espitia, R. E.; Carballo, C. A.; Zepeda, B. R.; Vaquera, H. H. y Córdova, T. L. 2011. Fertilización y densidad de plantas en variedades de amaranto (Amaranthus hypochondriacus L.). Rev. Mex. Cienc. Agríc. 2(6):855-866.

Rastogi, A. and Shukla, S. 2013. Amaranth: a new millennium crop of nutraceutical values. Critical Reviews in Food Science and Nutrition. 53(1):109-125. doi.org/10.1080/10408398.2010. 517876.

Rojas, W.; Pinto, M.; Alanoca, C.; Gómez, P. L.; Leónlobos, P.; Alercia, A.; Diulgheroff, S.; Padulosi, S. y Bazile, P. 2014. Conservación ex situ de los recursos genéticos de quinua. In: Bazile, D.; Bertero, D. y Nieto, C. (Ed.). Estado del arte de la quinua en el mundo en 2013. FAO. Santiago de Chile y CIRAD FAO. Santiago de Chile y CIRAD, Montpellier, Francia. 65-94 pp. https://agritrop.cirad.fr/574151/1/document_574151.pdf.

Thiam, E.; Alloui, A.; Benlhabib, O. 2021. Quinoa productivity and stability evaluation through varietal and environmental interaction. Plants. 10(714):1-14. doi.org/10.3390/plants 10040714.

Tiwari, S.; Pant, N. Ch.; Gupta, A.; Dwivedi, U.; Meena, J. K.; Pandey, C. S.; Dhoundiyal, R. and Bhatt, A. 2018. Genetic variability and genetic divergence for seed yield and its components characters in grain amaranth (Amaranhus hypochondriacus L.) germplasm. Inter. J. Chem Stud. 6(2):3292-3297.

Venskutonis, P. R. and Kraujalis, P. 2013. Nutritional components of amaranth seeds and vegetables: a review on composition, properties, and uses. Comprehensive Reviews in Food Science and Food Safety. 12(2):381-412. doi: 10.1111/1541-4337.12021.

Zurita-Silva, A.; Fuentes, F.; Jacobsen, S. E. and Schwember, A. 2014. Breeding quinoa (Chenopodium quinoa Willd.): potential and perspectives. Mol. Breed. 34(1):13-30. https://link.springer.com/article/10.1007/s11032-014-0023-5. 